\title{
Indicadores de Estresse Térmico e Utilização da Somatotropina Bovina (bST) em Vacas Leiteiras Mestiças (Bos taurus $x$ Bos indicus) no Semi-Árido do Nordeste ${ }^{1}$

\author{
Joaquim Batista de Oliveira Neto², Arlindo de Alencar Araripe Moura2, 3 ,
} José Neuman Miranda $\mathrm{Neiva}^{2}$, Magda Maria Guilhermino ${ }^{4}$
}

\begin{abstract}
RESUMO - O objetivo do presente experimento foi avaliar a resposta ao bST de vacas mestiças (Bos taurus $x$ Bos indicus) criadas no semi-árido do Ceará. Quinze vacas (segunda e terceira parições; 42 a 155 dias pós-parto) receberam cinco aplicações de bST a intervalos de 14 dias e outras 15 foram utilizadas como controle. A dieta dos animais consistiu de capim-elefante e concentrado. Durante a primeira aplicação de bST, a produção de leite dos animais tratados (12,6 kg/dia) não diferiu dos animais controle (11,3 Kg/dia) mas, na segunda e terceira aplicações, a diferença entre os grupos foi significativa $(2,0$ e 2,2 kg). Na quarta aplicação de bST, houve aumento na diferença entre os grupos (3,0 kg) e, na quinta aplicação, esta diferença foi de $3,4 \mathrm{~kg}(14,0 \mathrm{~kg}$ versus 10,6 kg/dia). A interação tratamento com bST e estágio de lactação não foi significativa. As temperaturas do leite e retal foram maiores $\left(0,6 \mathrm{e} 0,1^{\circ} \mathrm{C}\right.$, respectivamente $)$ nos animais tratados com bST que nos animais controles, mas estas diferenças não foram significativas. Para o grupo de 30 animais, houve correlações entre produção e temperatura do leite $(\mathrm{r}=0,38$ a 0,69). Com base no cálculo do índice de temperatura e umidade, os animais estiveram submetidos a estresse térmico ameno (manhã) ou moderado (à tarde). Portanto, animais mestiços criados no semi-árido do Nordeste aumentaram a produção de leite devido ao bST, sem, no entanto, apresentar indicativos de estresse térmico severo.
\end{abstract}

Palavras-chave: Bos indicus, estresse térmico, produção de leite, semi-árido, somatotropina

\section{Heat stress indexes and the Use of Bovine Somatotropin (bST) in Crossbred Dairy cows (Bos indicus $x$ Bos taurus) Raised in a Semi-Arid Region of the Brazilian Northeast}

\begin{abstract}
A study was conducted to evaluate the effects of bST in crossbred dairy cows (Bos indicus $x$ Bos taurus) raised in a semi-arid zone, in the Northeast of Brazil. Fifteen cows (second and third lactation; 42-155 days in milk) received five applications of bST, at 14-day intervals. Other 15 cows were used as controls. The animals were fed with diet consisted in elephant grass and concentrate. During the first application of bST, the milk production of the treated cows $(12.6 \mathrm{~kg} / \mathrm{day})$ did not differ from the control animals $(11.3 \mathrm{~kg} / \mathrm{day})$, but in the second and third applications, the difference between the groups was significant $(2.0 \mathrm{and} 2.3 \mathrm{~kg})$. In the fourth application of bST, there was an increase in the difference between groups $(3.0 \mathrm{~kg})$ and in the fifth application, such difference was $3.4 \mathrm{~kg}$ ( $14.0 \mathrm{~kg}$ versus $10.6 \mathrm{~kg} / \mathrm{day})$. The bST treatment and stage of lactation interaction was not significant. Rectal and milk temperatures were higher $\left(0.1\right.$ and $0.6{ }^{\circ} \mathrm{C}$, respectively) in bST treated than in control animals, but differences were not significant. For the group of 30 cows, the daily milk yield was related to milk temperature $(r=0.38$ to 0.69$)$. Based on the temperature-humidity index, the cows were under light heat stress in the morning and moderate stress in the afternoon. Therefore, crossbred cows raised in the semiarid area of Brazil showed responses to bST without apparent signs of severe heat stress.
\end{abstract}

Key Words: Bos indicus, Brazil, heat stress, milk yield, semi-arid, somatotropin

\section{Introdução}

A somatotropina bovina (bST) promove aumentos no consumo de matéria seca e na produção de leite, em função das condições de manejo alimentar e sanitário dos rebanhos (BAUMAN et al., 1985; HUBER et al., 1997). O uso do bST em vacas em lactação tem efeitos sobre a partição preferencial de nutrientes para a síntese de leite (TYRRELL et al., 1982), interferindo no metabolismo de vários órgãos e tecidos através de ações diretas (tecido adiposo e fígado) e efeitos indiretos mediados pelo IGF-I (BAUMAN, 1992).

Constata-se aumento na produção de calor em animais suplementados com bST, o qual é consequiência de alterações no metabolismo e produção de leite (TYRRELL et al., 1988). Vacas holandesas tratadas com bST e submetidas a temperaturas elevadas (27 a $40^{\circ} \mathrm{C}$ ) respondem com aumentos na produção de leite, mas apresentam valores mais elevados de

\footnotetext{
1 Parte da dissertação de mestrado do primeiro autor, apresentada à UFC. Apoio financeiro: Fundação de Amparo à Pesquisa do Estado do Ceará (FUNCAP).

2 Professor Adjunto - Departamento de Zootecnia - Universidade Federal do Ceará. Av. Mister Hull, s/n Campus do Pici - Fortaleza - CE - 60.021 - 970.

3 Correspondência. E-mail: amoura@ufc.br

4 Pesquisadora - Instituto de Zootecnia - Ribeirão Preto - SP.
} 
temperatura retal e freqüência respiratória. No entanto, o tratamento com bST não causou alterações nestes parâmetros fisiológicos, quando os animais foram mantidos em condições de termoneutralidade (COLE e HANSEN, 1993; ZOA-MBOE et al., 1989). Outros experimentos realizados na Florida e Georgia (EUA) mostraram também que acréscimos na temperatura ambiente estão associados a reduções na produção de leite em vacas tratadas com bST (ELVINGER et al., 1988; WEST, 1994). Portanto, o uso de bST pode predispor os animais à maior sensibilidade aos efeitos do estresse térmico, evidenciada pelas alterações na frequiência respiratória e temperatura retal. A produção de leite pode também ser influenciada por fatores climáticos, como temperatura ambiente e umidade relativa do ar, dependendo das características individuais de cada animal. A região semi-árida do Nordeste apresenta características únicas de clima no Brasil, mas as interações entre este tipo de ambiente e indicadores de estresse térmico em animais de produção têm sido pouco estudadas.

Animais mestiços (Holandês x Gir) representam grande porcentagem do rebanho leiteiro do Brasil. No Estado do Ceará, a produtividade média dos rebanhos é de 2,5 kg/vaca/dia (IBGE, 1997), mas existem, no entanto, várias propriedades com média de produção superior a 10-12 kg de leite/vaca/dia, devido à utilização de melhor tecnologia. Algumas dessas propriedades utilizam o bST, mas não há uma avaliação dos efeitos do emprego desta prática na realidade daqueles sistemas de produção localizados na região semiárida. Portanto, este estudo foi conduzido com o objetivo de avaliar as variações no índice de temperatura e umidade e o efeito do bST sobre a produção de leite, temperatura retal e temperatura do leite em vacas mestiças (Bos taurus $x$ Bos indicus) criadas em condições de manejo e ambiente característicos de uma propriedade localizada no semi-árido do Nordeste.

\section{Material e Métodos}

\section{Local e instalação do experimento}

O experimento foi realizado na Fazenda Caraíbas, município de Quixeramobim-CE, a 200 km de Fortaleza, com 78,0 metros de altitude na sede e coordenadas geográficas de $5^{\circ} 30^{\prime}$ de Latitude sul e $39^{\circ} 18^{\prime}$ de Longitude. A precipitação média é de 707,7 mm/ano, concentrada nos meses de fevereiro a maio (Fundação Cearense de Meteorologia, 1998). No período experimental (agosto a outubro de 1998), a fazenda produzia, em média, 1500 litros de leite/dia, com 125 vacas. A porcentagem de vacas em lactação era de $76 \%$ e o grau de sangue da maioria dos animais variava de $3 / 4$ a 7/8 Holandês x Gir, com poucos animais $1 / 2$ sangue. A fazenda contava com assistência periódica de um médico veterinário e realizava inseminação artificial. O proprietário possuía uma unidade de pausteurização com capacidade para $5000 \mathrm{l} / \mathrm{dia}$, onde era beneficiado todo o leite da fazenda, além de cotas adquiridas de outros produtores da região.

Procedimento experimental

Foram utilizadas trinta vacas mestiças Bos indicus $x$ Bos taurus (3/4 a 7/8 Holandês x Gir), entre a segunda e terceira parição, com 42 a 155 dias pósparto. A duração do experimento foi de 84 dias, sendo 14 dias de adaptação e 70 dias de período experimental. Após o período de adaptação, um grupo de 15 vacas recebeu cinco aplicações de $500 \mathrm{mg}$ de bST (Monsanto, St. Louis, MO, USA) a intervalos de 14 dias, por via parenteral subcutânea, alternando-se os lados a cada aplicação. O grupo controle também foi formado por 15 animais e não recebeu tratamento algum. Para a formação destes grupos, procurou-se manter uniformidade quanto à idade dos animais, nível de produção, estágio de lactação e ordem de parição. O bST foi aplicado entre 7 e $8 \mathrm{~h}$, logo após a pesagem do leite da manhã. Os grupos foram confinados em um curral de $1350 \mathrm{~m}^{2}$, com livre acesso a áreas de sombra e sol, com comedouros, bebedouros e cocho para mistura mineral.

As áreas de capim-elefante foram adubadas de acordo com as análises de solo e os cortes das forrageiras programados para intervalos entre $45 \mathrm{e}$ 52 dias. Os animais de ambos os grupos receberam capim-elefante picado fornecido à vontade, permitindo sobras de $10 \%$. O concentrado foi fornecido de acordo com a produção de leite, conforme manejo já adotado na fazenda. Com base no consumo durante a fase de adaptação, no peso vivo e na produção de leite de cada vaca, a dieta dos animais foi calculada (NATIONAL RESEARCHCOUNCIL - NRC, 1989). As exigências nutricionais não atendidas pelo volumoso foram corrigidas com a utilização de concentrado (22\% de proteína bruta, $78 \%$ de nutrientes digestíveis totais), à base de milho $(69,2 \%)$, farelo de soja $(23,9 \%)$, calcário $(1,4 \%)$, fosfato bicálcico $(2,4)$, sal comum $(0,8 \%)$, uréia $(1,8 \%)$, sulfato de amônia $(0,8 \%)$ e premix vitamínico $(0,1 \%)$ e mineral $(0,1 \%)$. Em média, cada vaca recebeu $1 \mathrm{~kg}$ de concentrado para 
No Brasil, FONTES et al. (1997), suplementando vacas mestiças (Bos indicus x Bos taurus) com bST, entre 55 e 199 dias de lactação, obtiveram resposta no início do tratamento de $2,5 \mathrm{~kg} / \mathrm{dia}$, com aumentos de produção nas aplicações seguintes de até $3,3 \mathrm{~kg} / \mathrm{dia}$, valores estes semelhantes aos descritos no presente estudo. Também no Brasil, SUÁREZ LONDOÑO et al. (1997), suplementando com bST por 140 dias vacas mestiças (Bos indicus x Bos taurus) e holandesas, obtiveram incremento médio na produção de leite de $1,5 \mathrm{~kg} / \mathrm{vaca} /$ dia para animais confinados e apenas $0,6 \mathrm{~kg} / \mathrm{vaca} /$ dia para animais semi-confinados, sendo a menor resposta do grupo semi-confinado devido ao tipo de volumoso fornecido (capim gordura e capim-elefante picado). PEEL et al. (1987) obtiveram consistentes aumentos na produção de leite ( 2 a $5 \mathrm{~kg}$ / vaca/dia) em vacas Holandesas tratadas com bST no início, meio e final da lactação. Segundo CHALUPA et al. (1989), animais suplementados com bST tiveram produção de leite $40 \%$ acima do grupo controle, com grande variação de resposta relacionada à quantidade da dose de bST, freqüência de aplicações, manejo e outros fatores. PHIPPS et al. (1991) observaram aumentos na produção de leite de 3,$5 ; 3,4$; e $3,2 \mathrm{~kg} / \mathrm{dia}$ em vacas holandesas tratadas com bST aos 90, 120 e 150 dias pós-parto, em diferentes rebanhos na África. Respostas maiores $(6,1 \mathrm{~kg} / \mathrm{vaca} / \mathrm{dia})$ foram também observadas em alguns destes rebanhos, devido ao excelente planejamento alimentar e tardio início do tratamento.
Apesar de a alimentação dos animais ter sido planejada de acordo com as normas do NRC (1989), mudanças no sistema de manejo da fazenda durante o experimento causaram atrasos nos períodos de corte do capim elefante e, consequientemente, as plantas foram cortadas com idade acima dos 90 dias. A ausência de resposta na primeira aplicação e diminuição da produção na terceira aplicação de bST são provavelmente consequiências destes eventos. Nos períodos associados à quarta e quinta aplicação de bST, as forrageiras voltaram a ser cortadas com 45 a 52 dias de idade, coincidente com maiores respostas das vacas ao bST. Segundo BAUMANN et al. (1985), animais suplementados com bST devem ter disponibilidade de alimentos de boa qualidade durante o maior tempo possível e o nível de manejo é a principal influência na resposta da produção.

A ordem de parição e o grau de sangue não representaram fontes significativas de variação na resposta dos animais ao tratamento com bST. De modo semelhante, REMOND et al. (1991) também mostraram que ordem de parição não está associada à resposta das vacas ao bST e FONTES et al. (1997) verificaram que o grau de sangue (1/2 versus $3 / 4$ Bos taurus x Bos indicus) não teve influência sobre o tratamento com bST. Foi avaliada também a diferença de resposta ao bST em relação ao estágio de lactação, dividindo-se os grupos de animais em dois subgrupos menores, sendo um com 42 a 100 dias de lactação e o outro com 101 a 155 dias de lactação (Tabela 2). Na

Tabela 2 - Efeito da somatotropina bovina (bST) na produção de leite (kg/dia) de vacas mestiças (Bos indicus x Bos taurus), segundo estágio de lactação, no semi-árido do Nordeste

Table 2 - Effects of bovine somatotropin (bST) on milk yield ( $\mathrm{kg} /$ day), according to stage of lactation, in crossbred cows raised in the semiarid region in the Brazilian Northeast

\begin{tabular}{|c|c|c|c|c|}
\hline \multirow[b]{2}{*}{$\begin{array}{l}\text { Aplicações de bST } \\
\text { BST treatments }\end{array}$} & \multicolumn{2}{|c|}{$\begin{array}{l}\text { 42-100 dias de lactação }{ }^{1} \\
42-100 \text { days in milk }\end{array}$} & \multicolumn{2}{|c|}{$\begin{array}{l}\text { 101-144 dias de lactação } \\
101 \text { - } 144 \text { days in milk }\end{array}$} \\
\hline & $\begin{array}{l}\text { Controle } \\
(\mathrm{n}=8)^{2}\end{array}$ & $\begin{array}{c}\mathrm{bST} \\
(\mathrm{n}=10)^{2}\end{array}$ & $\begin{array}{l}\text { Controle } \\
(\mathrm{n}=7)^{2}\end{array}$ & $\begin{array}{c}\mathrm{bST} \\
(\mathrm{n}=5)^{2}\end{array}$ \\
\hline 1 & $12,4 \pm 1,15^{\mathrm{a}}$ & $13,0 \pm 1,05^{\mathrm{a}}$ & $10,1 \pm 1,13^{\mathrm{a}}$ & $11,8 \pm 1,48^{a}$ \\
\hline 2 & $12,0 \pm 0,97^{\mathrm{a}}$ & $13,7 \pm 1,11^{\mathrm{a}}$ & $10,4 \pm 1,03^{\mathrm{a}}$ & $12,3 \pm 1,28^{\mathrm{a}}$ \\
\hline 3 & $11,5 \pm 0,86^{\mathrm{a}}$ & $12,9 \pm 1,01^{\mathrm{a}}$ & $9,5 \pm 0,75^{\mathrm{a}}$ & $11,8 \pm 1,21^{\mathrm{a}}$ \\
\hline 4 & $12,1 \pm 1,17^{\mathrm{a}}$ & $14,4 \pm 1,13^{\mathrm{a}}$ & $10,2 \pm 0,93^{a}$ & $13,7 \pm 1,21^{\mathrm{a}}$ \\
\hline 5 & $12,0 \pm 1,00^{\mathrm{a}}$ & $14,3 \pm 1,10^{\mathrm{a}}$ & $10,4 \pm 1,10^{\mathrm{a}}$ & $13,8 \pm 1,20^{\mathrm{a}}$ \\
\hline \multicolumn{5}{|l|}{ Average } \\
\hline $\begin{array}{ll}1 & \text { Valores representan } \\
2 & \text { Número de observa } \\
\text { a,b } & \text { Para cada período d } \\
1 & \text { Values represent the a } \\
2 & \text { Number of observations } \\
\text { a,b } & \text { Within }\end{array}$ & $\begin{array}{l}\text { respectivos err } \\
\text { bST e estágio } \\
\text { espective standar }\end{array}$ & ão há diferença & ara médias com & letras $(P>0,05)$. \\
\hline
\end{tabular}


364 Rev. bras. zootec.

quarta e quinta aplicações, a diferença entre os tratamentos foi de $2,3 \mathrm{~kg}$ para os animais em início de lactação e 3,5 kg de leite para animais com 101-155 dias de lactação. Provavelmente, devido ao pequeno número de animais em cada subgrupo, estas diferenças não foram significativas ( $\mathrm{P}>0,05)$. Portanto, animais $3 / 4$ e 7/8 Holandês x Gir possuem características diferentes daquelas observadas em animais $1 / 2$ sangue. Tem sido mostrado que em vacas $1 / 2$ Bos taurus $\mathrm{x} 1 / 2$ Bos indicus as respostas são reduzidas após os 100 dias de lactação (FONTES et al., 1997), enquanto vacas Bos taurus apresentam maior resposta ao tratamento com bST no final da lactação (THOMAS et al., 1991).

\section{Parâmetros fisiológicos (temperatura do leite e temperatura retal) e ambiente}

A temperatura retal não foi influenciada pelo bST $(\mathrm{P}>0,05)$. Em média, os animais tratados apresentaram temperatura retal de $38,7 \pm 0,04^{\circ} \mathrm{C}$ pela manhã e $38,9 \pm 0,04^{\circ} \mathrm{C}$ à tarde e os animais do grupo controle, $38,7 \pm 0,03^{\circ} \mathrm{C}$ pela manhã e $38,8^{\circ} \mathrm{C} \pm 0,04$ à tarde (Figura 1). A diferença de temperatura retal entre os períodos da manhã e tarde foi de $0,2^{\circ} \mathrm{C}$ para as vacas que receberam bST e $0,1^{\circ} \mathrm{C}$ para o grupo controle

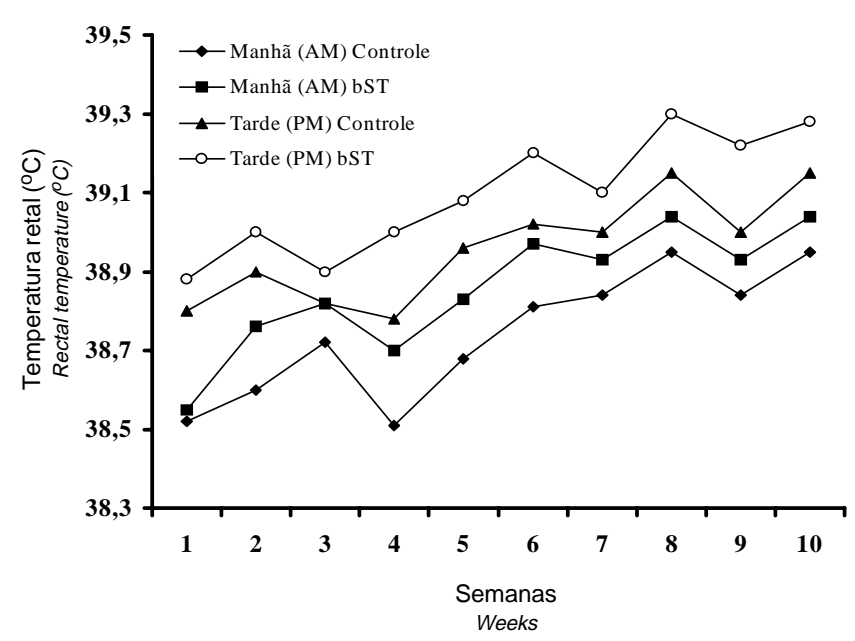

Figura 1 - Temperatura retal em vacas mestiças (Bos taurus $x$ Bos indicus) tratadas e não tratadas com bST no semi-árido do Nordeste. Os animais receberam doses de bST a cada 14 dias, a partir da semana 1. Não houve diferença significativa entre os grupos para os períodos manhã e tarde $(P>0,05)$.

Figure 1 - Rectal temperature in crossbred dairy cows (Bos taurus $x$ Bos indicus) treated or not with bST, in a semi-arid region of the Brazilian Northeast. Animals received bST at 14-day intervals, starting form week 1. There were no significant differences between groups in the morning and afternoon $(P>.05)$.
( $P>0,05$; Figura 1). Em concordância com os resultados acima, ZOA-MBOE et al. (1989), trabalhando com vacas holandesas no Estado da Georgia (USA), detectaram aumento da temperatura retal $(39,99$ versus $39,81^{\circ} \mathrm{C}$ ), devido ao tratamento com bST, em ambiente com temperaturas de 21,2 e $32,7^{\circ} \mathrm{C}$ e umidade relativa do ar (UR) de 50 a $90 \%$. No entanto, esta diferença de $0,1^{\circ} \mathrm{C}$ foi significativa. ELVINGER et al. (1992) também encontraram aumentos de $0,1^{\circ} \mathrm{C}$ na temperatura retal em animais tratados com bST, sob temperaturas entre 27 e $33^{\circ} \mathrm{C}$ e umidade relativa de 77 a $85 \%$, embora esta variação de $0,1^{\circ} \mathrm{C}$ não tenha sido significativa $(\mathrm{P}>0,05)$. Nesses estudos, os resultados das análises estatísticas foram diferentes para a mesma variação de temperatura retal $\left(0,1^{\circ} \mathrm{C}\right)$ encontrada nos grupos de vacas do presente experimento. A razão destas diferenças não é clara mas pode está relacionada a variações na homogeneidade dos grupos dos animais avaliados nos diferentes ambientes.

Estudos têm mostrado que condições de estresse térmico mais severas apresentam efeitos significativos sobre os animais tratados com bST. COLE e HANSEN (1993), trabalhando com vacas holandesas submetidas a temperaturas de 33 a $40^{\circ} \mathrm{C}$ e umidade relativa de 78 a $90 \%$, observaram aumentos $(\mathrm{P}<0,01)$ na temperatura retal dos animais tratados com bST que variaram de 0,3 a $0,4^{\circ} \mathrm{C}$. ELVINGER et al. (1992), trabalhando com 34 vacas holandesas sob temperaturas de 37 a $43^{\circ} \mathrm{C}$ e umidade relativa de 85 a $95 \%$, verificaram aumentos de $0,6^{\circ} \mathrm{C}(\mathrm{P}<0,01)$ na temperatura retal devido ao uso do bST.

Os animais que receberam bST apresentaram valores mais elevados de temperatura do leite do que os animais controle pela manhã $\left(35,5 \pm 0,29^{\circ} \mathrm{C}\right.$ versus 35,1 $\left.\pm 0,10^{\circ} \mathrm{C}\right)$ e à tarde $\left(36,8 \pm 0,23^{\circ} \mathrm{C}\right.$ versus $\left.36,2 \pm 0,15^{\circ} \mathrm{C}\right)$, embora estas diferenças de 0,4 e $0,6^{\circ} \mathrm{C}$ não tenham sido significativas $(\mathrm{P}>0,05$, Figura 2$)$. Dentro de cada grupo de animais, a temperatura do leite apresentou aumentos significativos $(\mathrm{P}<0,05)$ entre os períodos da manhã e da tarde, com diferenças médias de $1,3^{\circ} \mathrm{C}$ para os animais tratados e $1,1^{\circ} \mathrm{C}$ para o grupo controle. WEST et al. (1991) observaram que vacas Holandesas $(n=6)$ e Jersey $(n=8)$ tratadas com bST e mantidas em temperaturas de 22 a $35^{\circ} \mathrm{C}(72 \%$ UR) apresentaram aumentos $(\mathrm{P}<0,01)$ de 0,3 a $0,6^{\circ} \mathrm{C}$ na temperatura do leite em relação aos grupos controle. Dado que o número de animais no citado experimento foi semelhante ao utilizado no presente trabalho, a uniformidade do rebanho e, conseqüentemente, menor variabilidade dentro dos grupos tratados e controle provavelmente influenciaram os resultados dos testes estatísticos. 
Uma vez que não houve diferença entre os grupos para temperatura do leite, foram realizadas análises de correlação com todos os 30 animais do experimento. Houve correlações entre a produção diária de leite e a temperatura do leite pela manhã e à tarde $(r=0,38$ a 0,$69 ; \mathrm{P}<0,05$; Tabela 3). Sabe-se que vacas com maior produção estão mais sujeitas a efeitos do ambiente e variações na temperatura do leite estão associadas a maiores produções (WEST et al., 1991). Os resultados apresentados nesta pesquisa mostram a mesma tendência descrita por outros autores, ou seja, que há uma associação entre aumento da produção e temperatura do leite. De acordo com a literatura consultada, este experimento é o primeiro a mostrar correlações entre produção e temperatura do leite em vacas criadas no semi-árido do Nordeste. A razão da existência desta correlação não é clara, mas, provavelmente, o bST não provocou alteração no metabolismo dos animais a ponto de causar aumento substancial na temperatura retal. No entanto, variações na temperatura do leite estiveram mais associadas à produção, sugerindo que a glândula mamária

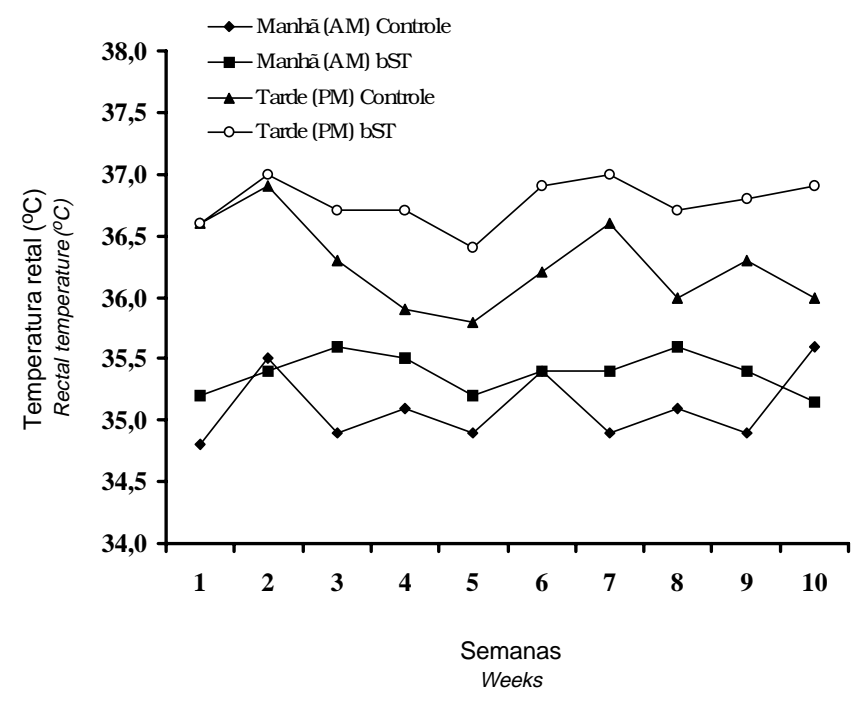

Figura 2 - Temperatura do leite em vacas mestiças (Bos taurus $x$ Bos indicus) tratadas e não tratadas com bST no semi-árido do Nordeste. Os animais receberam doses de bST a cada 14 dias, a partir da semana 1. Não houve diferença significativa entre os grupos para os períodos manhã e tarde $(P>0,05)$.

Figure 2 - Milk temperature in cros-bred dairy cows (Bos taurus $x$ Bos indicus) treated or not with bST, in a semi-arid region of the Brazilian Northeast. Animals received bSTat 14-dayintervals, starting form week 1 . There were no significant differences between groups in the morning and afternoon (P>.05). pode ter maior dificuldade de dissipar o calor, devido à localização na cavidade inguinal e intenso fluxo sangüíneo. As correlações entre temperatura do leite e produção podem indicar que, em vacas com maior produção de leite, há a possibilidade de estresse térmico mais severo.

Na propriedade onde foi realizado o experimento, as médias de temperatura máxima e mínima foram 37,7 e $20,1^{\circ} \mathrm{C}$, respectivamente, com variações de umidade relativa do ar entre 48 e $70 \%$ (Tabela 4). Com a avaliação do índice de temperatura e umidade (ITU), no período da manhã, verificou-se que os animais estiveram submetidos a estresse ameno, com temperatura ambiente e umidade relativa média de $26,8^{\circ} \mathrm{C}$ e $61 \%$, respectivamente (Figura 3 ). À tarde, no período de 14 a 25 de agosto, houve estresse ameno e durante o restante do período, estresse moderado, com temperatura média e umidade relativa de $33,2^{\circ} \mathrm{C}$ e $48 \%$, respectivamente. Foi detectado, durante o período experimental, um ponto máximo com estresse severo durante apenas uma tarde $($ ITU $=90)$, devido principalmente a mudanças na

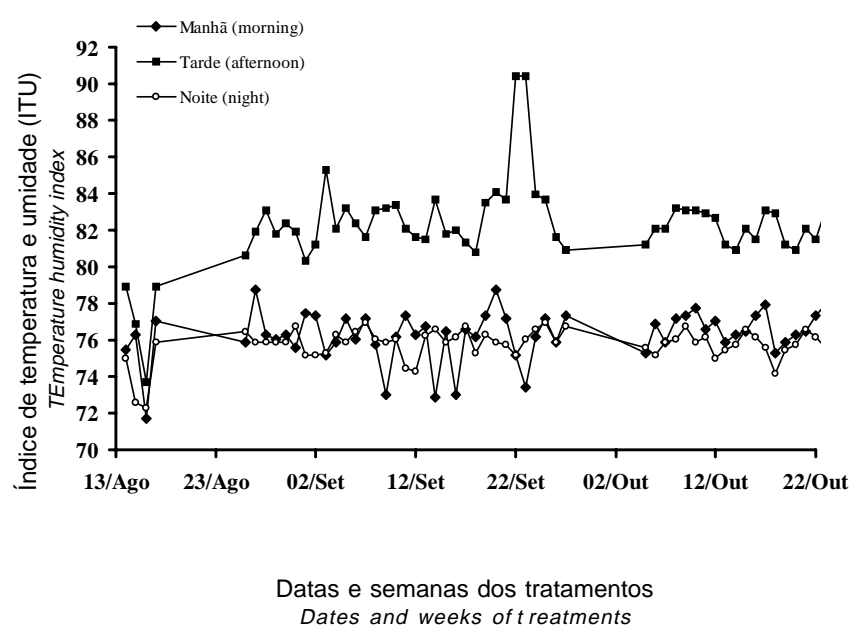

Figura 3 - Índice de temperatura e umidade (ITU) avaliado em estábulo coberto, no município de Quixeramobim, semi-árido do Nordeste, durante os meses de agosto a outubro.

Figure 3 - Temperature-humidity index evaluated in a shaded barn, in the county of Quixeramobim, semi-arid region of the Brazilian Northeast, from August to October. 
366 Rev. bras. zootec.

Tabela 3 - Correlações de Pearson entre produção de leite e temperatura do leite em vacas mestiças (Bos indicus x Bos taurus) na região semi-árida do Nordeste

Table 3 - Pearson correlations between milk yield and milk temperature in crossbred cows (Bos indicus $x$ Bos taurus) raised in the semiarid region of the Brazilian Northeast

\begin{tabular}{|c|c|c|c|c|c|}
\hline \multirow{2}{*}{$\begin{array}{l}\text { Aplicação de bST } \\
\text { bST treatment } \\
0^{1}\end{array}$} & \multirow{2}{*}{$\begin{array}{c}\text { Semana } \\
\text { Week }\end{array}$} & \multicolumn{2}{|c|}{$\begin{array}{l}\text { Manhã } \\
\text { Morning }\end{array}$} & \multicolumn{2}{|c|}{$\begin{array}{c}\text { Tarde } \\
\text { Afternoon }\end{array}$} \\
\hline & & $0,38^{* *}$ & $(\mathrm{n}=30)^{2}$ & $0,43^{* *}$ & $(\mathrm{n}=30)^{2}$ \\
\hline $0^{1}$ & 2 & $\mathrm{NS}^{1}$ & $(n=29)$ & $0,41^{* *}$ & $(n=29)$ \\
\hline 1 & 3 & $0,46^{* * *}$ & $(n=14)$ & $0,51^{* * *}$ & $(n=14)$ \\
\hline 1 & 4 & $0,57^{*}$ & $(\mathrm{n}=24)$ & $0,51^{*}$ & $(n=25)$ \\
\hline 2 & 5 & $0,54^{*}$ & $(\mathrm{n}=30)$ & $0,44^{* *}$ & $(n=30)$ \\
\hline 2 & 6 & $0,63^{*}$ & $(n=30)$ & $0,37^{* *}$ & $(n=30)$ \\
\hline 3 & 7 & $\mathrm{NS}^{1}$ & $(n=30)$ & $0,65^{*}$ & $(n=30)$ \\
\hline 3 & 8 & $0,60^{*}$ & $(\mathrm{n}=30)$ & $0,68^{*}$ & $(\mathrm{n}=30)$ \\
\hline 4 & 9 & $0,51^{*}$ & $(\mathrm{n}=18)$ & $0,56^{*}$ & $(\mathrm{n}=30)$ \\
\hline 4 & 10 & $0,46^{* *}$ & $(\mathrm{n}=23)$ & $0,69^{*}$ & $(\mathrm{n}=23)$ \\
\hline 5 & 11 & $0,59^{*}$ & $(n=30)$ & $0,68^{*}$ & $(n=30)$ \\
\hline 5 & 12 & $0,49^{*}$ & $(\mathrm{n}=30)$ & $0,67^{*}$ & $(\mathrm{n}=28)$ \\
\hline
\end{tabular}

Pré-tratamento.

2 Número de observações.

NS: não-significante $(P>0,10) .{ }^{*}(P<0,01) . ;{ }^{* *}(P<0,05) . ;{ }^{* \star *}(P<0,10)$.

1 Pre-treatment.

2 Number of observations.

NS: non-significant $(P>$.10).

Tabela 4 - Médias dos parâmetros climáticos avaliados durante a fase experimental, em estábulo coberto, no semi-árido do Nordeste

Table 4 - Average of climate indexes during the experimental period, evaluated in a barn with shade, in the semi-arid region of the Brazilian Northeast

\begin{tabular}{lccc}
\hline & $\begin{array}{c}\text { Manhã }(9 \mathrm{~h}) \\
\text { Morning }\end{array}$ & $\begin{array}{c}\text { Tarde }(15 \mathrm{~h}) \\
\text { Afternoon }\end{array}$ & $\begin{array}{c}\text { Noite }(21 \mathrm{~h}) \\
\text { Night }\end{array}$ \\
\hline $\begin{array}{l}\text { Temperatura máxima }\left({ }^{\circ} \mathrm{C}\right) \\
\text { Maximum temperature }\end{array}$ & 32,9 & 37,7 & 31,5 \\
Temperatura mínima $\left({ }^{\circ} \mathrm{C}\right)$ & 22,6 & 28,5 & 20,1 \\
$\begin{array}{l}\text { Minimum temperature } \\
\text { Temperatura bulbo seco }\left({ }^{\circ} \mathrm{C}\right)\end{array}$ & 26,8 & 33,2 & 26,7 \\
$\begin{array}{l}\text { Dry bulb temperature } \\
\text { Umidade relativa }(\%)\end{array}$ & 61 & 48 & 70 \\
Humidity & & & \\
\hline
\end{tabular}

umidade relativa do ar. À noite, as temperaturas reduzidas evitaram aumentos do estresse térmico para os animais (Figura 3). Apesar das altas temperaturas ambientais durante o período da tarde (temperatura máxima de $37,7^{\circ} \mathrm{C}$ ) durante o experimento, esta condição foi amenizada pela baixa umidade (48\%). A variação do consumo de matéria seca entre o dia e a noite não foi avaliada neste experimento. No entanto, as alterações observadas no Índice de Temperatura e Umidade podem sugerir que houve algum tipo de adaptação dos animais com relação ao hábito de consumo. SCHENEIDER et al. (1984) observa- ram que vacas mantidas sob estresse térmico consomem mais alimento durante as noite frias do que no decorrer do dia. O mesmo comportamento foi citado por PIRES et al. (1998), observando alteração no padrão de alimentação das vacas, com as mesmas alimentando-se mais durante o período noturno, evitando assim a ingestão de alimentos entre as $6 \mathrm{~h} 30$ e $17 \mathrm{~h}$.

\section{Conclusões}

Vacas Bos taurus x Bos indicus (3/4 a $7 / 8$ Holandês x Gir) apresentam respostas significativas 
ao tratamento com bST exógeno, sem, no entanto, ocorrerem alterações nos indicadores de estresse térmico avaliados (temperatura retal e do leite).

As condições de temperatura e umidade relativa do ar em local sombreado, no semi-árido, não apresentam limitações ao conforto e produção dos animais.

\section{Referências Bibliográficas}

ANUÁRIO ESTATÍSTICO DO BRASIL. Rio de Janeiro, IBGE, 1997.

BAUMAN, D.E., EPPARD, P.J., GEETER, M.J. et al. 1985. Response of high producing dairy cows to long term treatment with pipitultary and recombinant somatotropin. J. Dairy Sci. 68:1352-1368.

BAUMAN, D.E. 1992. Bovine somatotropin: review of na emerging animal technology. J. Dairy Sci., 75:3432-3451.

CHALUPA, W., GALligAN, D.T. 1989. Nutritional implications of somatotropin for lactating cows. J. Dairy Sci., 72:2510-2524.

COLE, J.A ., HANSEN, P.J. 1995. Effects of administration of recombinant bovine somatotropin on the responses of lactating and nonlactating cows to heat stress. JAVMA, 203(1): 113-117.

DAHL, G.E., CHAPIN, L.T., MOSELEY, W.M. et al. 1993. Galactopoietic effect of recombinant somatotropin and growth hormone-releasing factor in dairy cows. J. Dairy Sci., 76:1150-1557.

ELVINGER, F., HEAD, H.H., WILCOX, C.J. et al. 1988. Effects od administration of bovine somatotropin on milk yield and composition. J. Dairy. Sci., 71:1515.

ELVINGER, F., NATZKE, R.P., EGGERT, R.G. 1992. Interactions of heat stress and bovine somatotropin affecting physiology and immunology of lactating cows. J. Dairy Sci., 75:449-462.

FONTES JR., C., MESEROLE, V.K., MATTOS, W. et al. 1997. Response of brazilian crossbred cows to varying doses of bovine somatotropin. J. Dairy Sci., 80:3234-3240.

FUNDAÇÃO CEARENSE DE METEORÓLOGIA (FUCEME). 1998. In: Dados Climáticos, Fortaleza-CE. Secretária de Agricultura, Reforma Agrária e Abastecimento.

HUBER, J.T., WU,Z.C., FONTES JR., C. et al. 1997. Administration of recombinant bovine somatotropin to dairy cows for four consecutive lactations. J. Dairy Sci., 80:2355-2360.

NATIONAL RESEARCH COUNCIL - NRC. 1988. Nutrient requeriments of dairy cattle. 6 ed. Washington D.C.: National Academic of Sciences. p.158-162.

PEEL, C.J., FRONK, T.J., BAUMAN, D.E. et al. 1987. Effect of exogenous growth hormone in early and late lactation on lactational performance of dairy cows. J. Dairy Sci., 66:776-782.

PHIPPS, R.H., MADAKADZE, C., MUTSVANGWA, T. et al. 1991. Use of bovine somatrotopin in the tropics: the effect of sometribove on milk production of Bos indicus, dairy crossbred and Bos taurus cows in Zimbabwe. J. Dairy Sci., 76:177:257.
PIRES, M.F.A, CAMPOS, A.T., FERREIRA, A.M. Importância do conforto, ambiente e instalações no manejo de matrizes leiteiras. In: SIMPÓSIO - O AGRONEGÓCIO DO LEITE NO NORDESTE: ALTERNATIVAS TECNOLÓGIAS E PERSPECTIVAS DE MERCADO, 1988, Natal. Anais.... Natal, 1998. p.266-282.

REMOND, M.C., OLLIER, A., CHILLARD, Y. 1991. Slow release somatotropin in dairy heifers and cows fed two levels of energy concentrate. 1. Performance and body condition. J. Dairy Sci., 74:1370-1381.

SAS INSTITUTE. Statistical analysis systems user's guide: version 6, 4.ed., Cary, NC - SAS Institute, 1997. v.2.

SCHNEIDER, P. L., BREED, D. K., WILCOX, C. J. et al. 1984. Influence of dietary sodium and potassium bicarbonate and total potassium on heat-stressed lactating cows. J. Dairy Sci., 67: 2546-2557.

SUÀREZ LONDOÑO, A.A., VALADARES FILHO, S.C., COELHO DA SILVA, J. F. et al. 1997. Somatotropina bovina para vacas de leite em lactação. 1. Produção e composição do leite. J. Dairy Sci., 26:1227-1233.

THOMAS, J.W., ERDMAN, R.A., GALTON, D.M. 1991. Responses by lactating cows in commercial dairy herds to recombinant bovine somatotropin. J. Dairy Sci., 74:945-964.

TYRRELL, H.F., BROWN, A.C.G., REYNOLDS, P.J. et al. Effect of growth hormone on utilization of energy by lactating Holtein cows. In: NINTH SYMPOSIUM ON ENERGY METABOLISM (A. Ekern and F. Sundstol, ed.) EEAP Publication, 1982. $\mathrm{n}^{\circ} 29$.

TYRRELL, H.F., BROWN, A.C.G., REYNOLDS, P.J. et al. 1988. Effect of bovine somatotropin on metabolism of lactating dairy cows: energy and nitrogen utilization as determined by respiration calorimetry. J. Nutr., 118:1024-1035.

WEST, J.W., MULLINIX, B.G., SANDIFER, T.G. 1991. Effects of bovine somatotropin on physiologic responses of lactating holstein and jersey cows during hot, humid weather. J. Dairy Sci., 74:840-851.

WEST, J. W. 1994. Interactions of energy and bovine somatotropin with heat stress. J. Dairy Sci., 77:2091-2102.

ZOA-MBOE, A., HEAD, H.H., BACHMAN, K.C. et al. 1989. Effects of bovine somatrotopin on milk yield and composition, dry matter intake, and some physiological functions of Holstein cows during heat stress. J. Dairy Sci., 72:907-916.

Recebido em: 07/06/00 Aceito em: 08/11/00 